Supporting Information

\title{
Combining mechanical High-Intensity Focused Ultrasound Ablation with Chemotherapy for Augmentation of Anticancer Immune Responses
}

\author{
Chao Li ${ }^{\mathrm{a}, 1}$, Yao Lu ${ }^{\mathrm{a}, 1}$, Lili Cheng ${ }^{\mathrm{a}, 1}$, Xiaoge Zhang ${ }^{\mathrm{a}}$, Jun Yue ${ }^{\mathrm{a},}$, Jie Liu $^{\mathrm{a},{ }^{*}}$ \\ a School of Biomedical Engineering, Sun Yat-sen University, Guangzhou, Guangdong 510006, \\ China \\ ${ }^{1}$ These authors contributed equally to this work. \\ *Corresponding to: liujie56@mail.sysu.edu.cn, yuejun3@mail.sysu.edu.cn
}


A
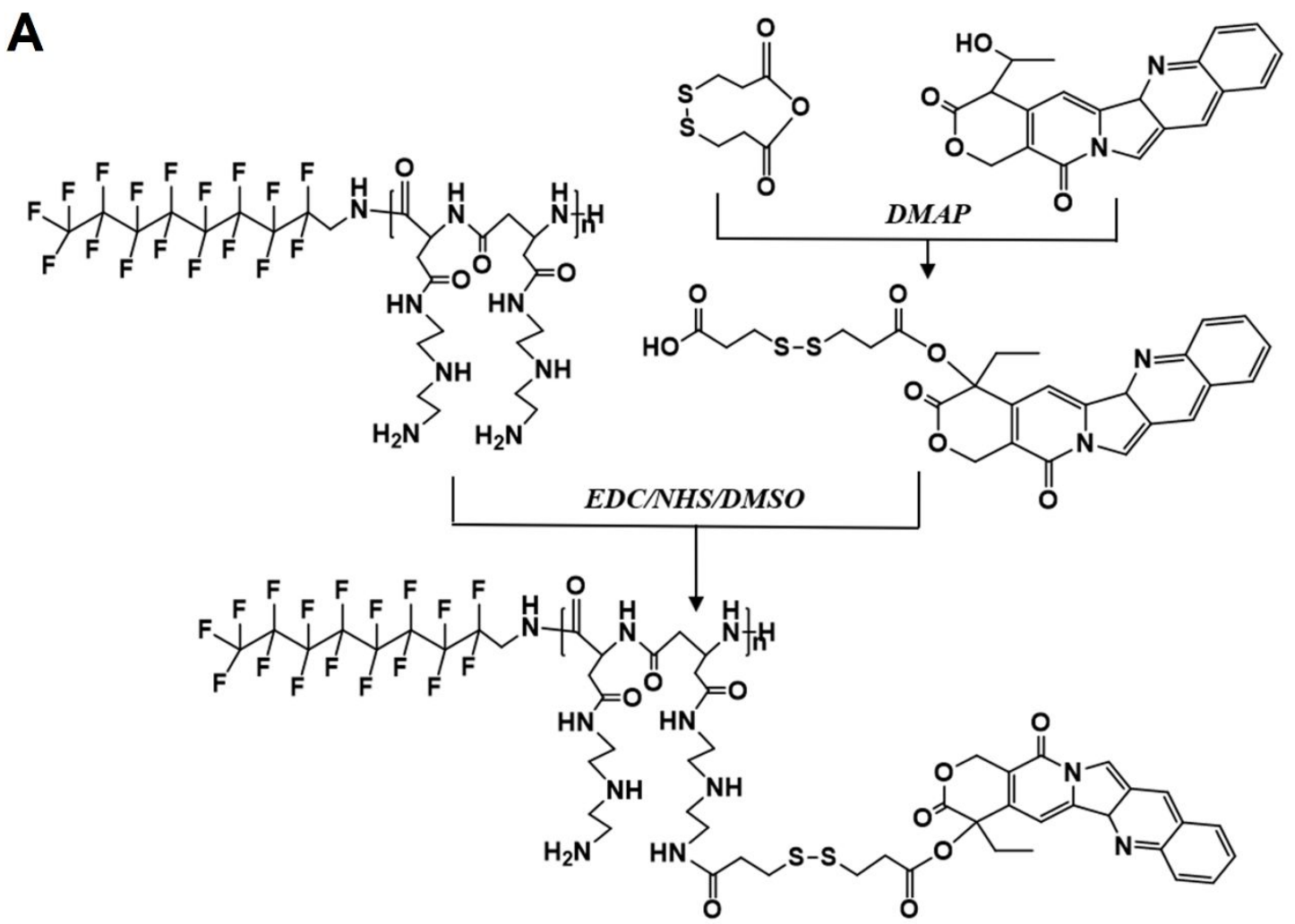

B
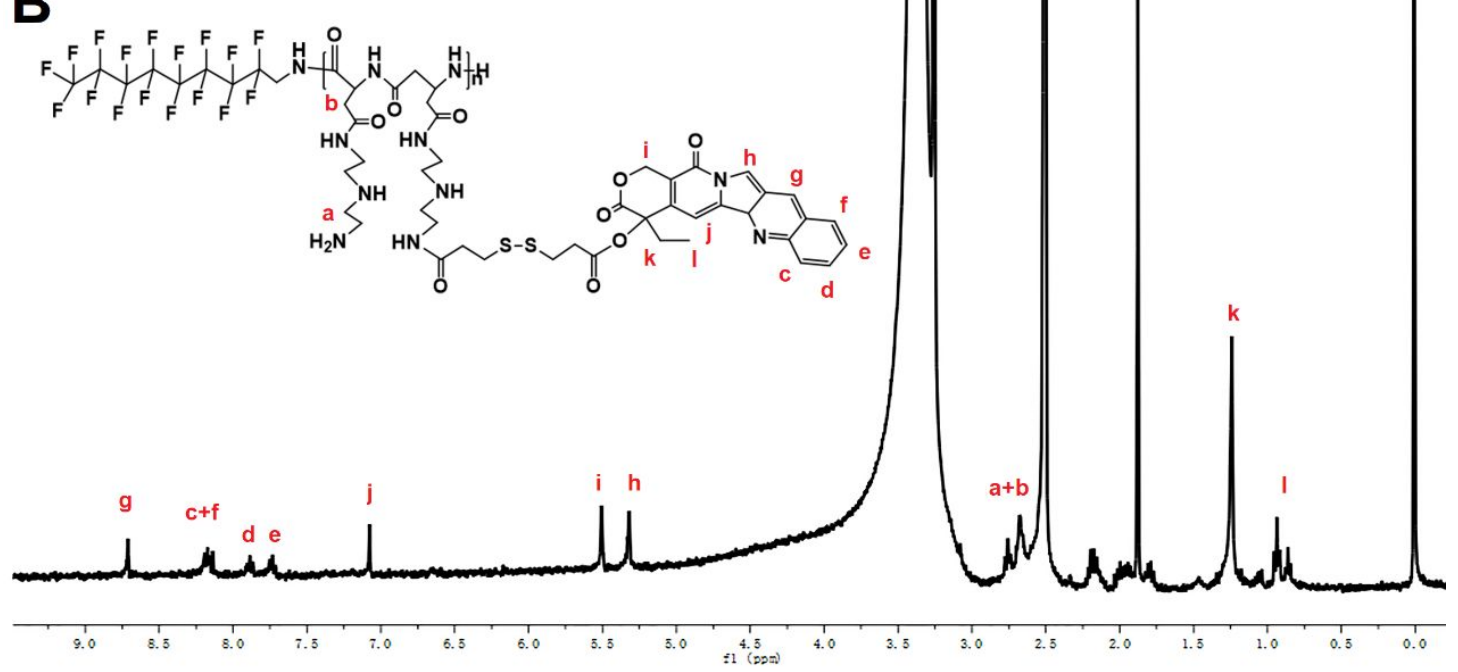

Figure S1. Synthesis route (A) and ${ }^{1} \mathrm{H}-\mathrm{NMR}$ spectrum (B) of polymer-drug conjugates containing disulfide linkages between CPT and amphiphilic PPCP copolymers $\left(\mathrm{C}_{9} \mathrm{~F}_{17^{-}}\right.$ PAsp(DET)-ss-CPT). The 1H-NMR spectrum of C9F17-PAsp(DET)-ss-CPT showed the appearance of peak at 8.08-8.20,7.89-7.71 and $7.18 \mathrm{ppm}$, which confirmed the successful conjugation of CPT with the amino groups of amphiphilic block copolymer. 


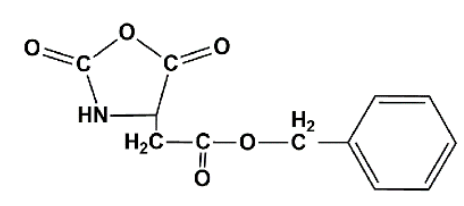

BLA-NCA
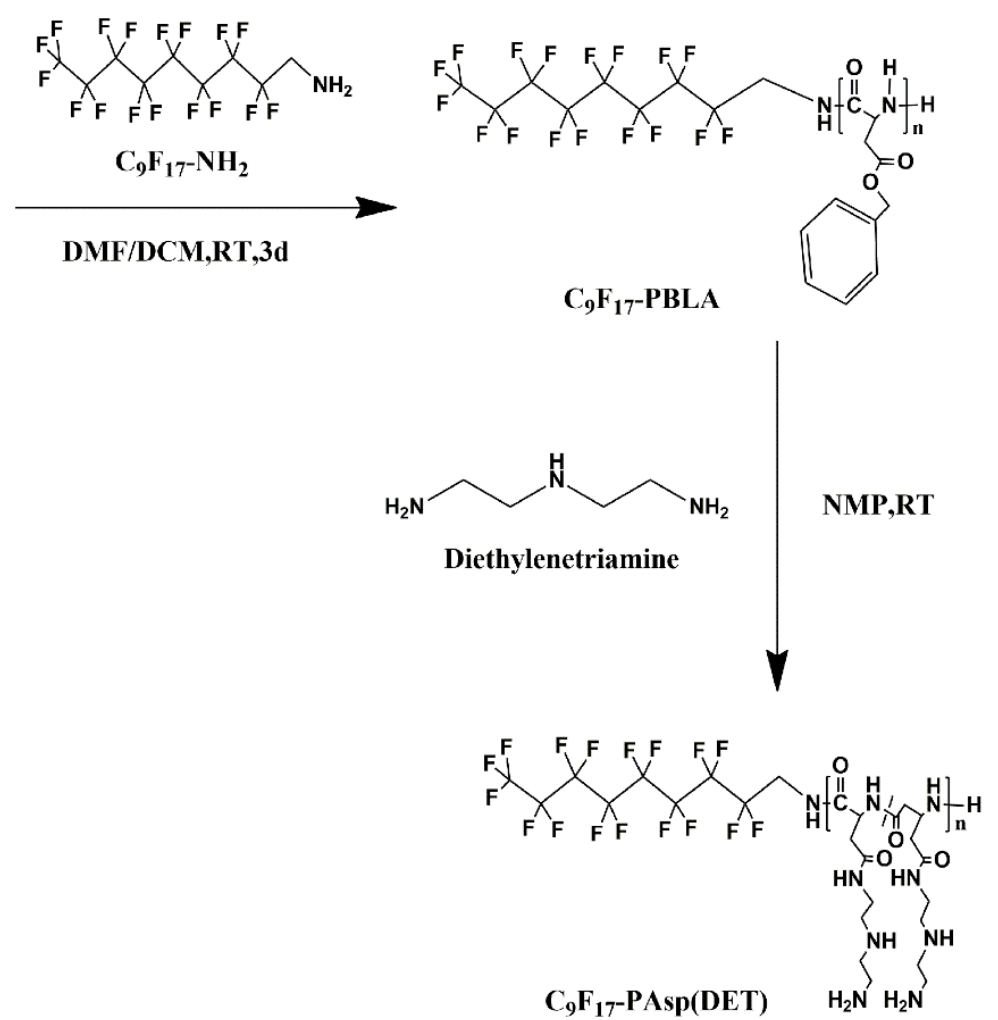

Scheme S1. Schematic of the synthesis of $\mathrm{C}_{9} \mathrm{~F}_{17}-\mathrm{PAsp}(\mathrm{DET})$. 


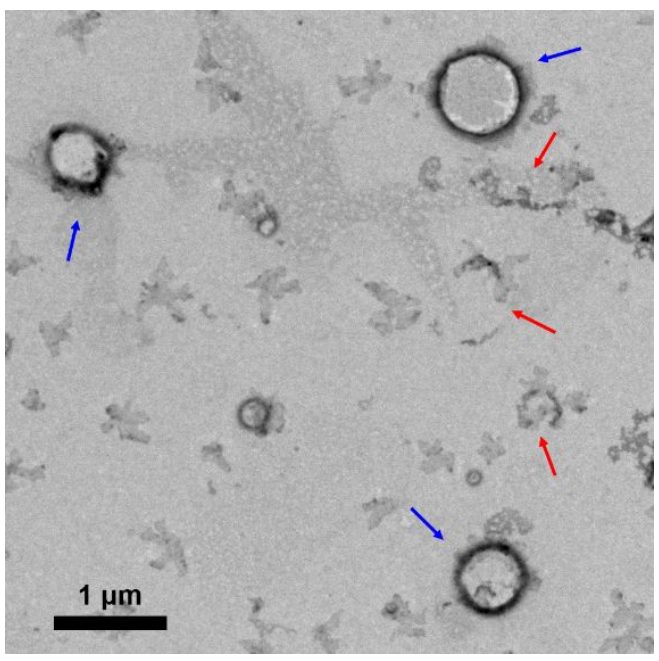

Figure S2. TEM images of PFP/CPT-codelivery systems after irradiation with HIFU under the condition of $3.5 \mathrm{MHz}, 20 \%$ duty cycle, $5 \mathrm{~W}$
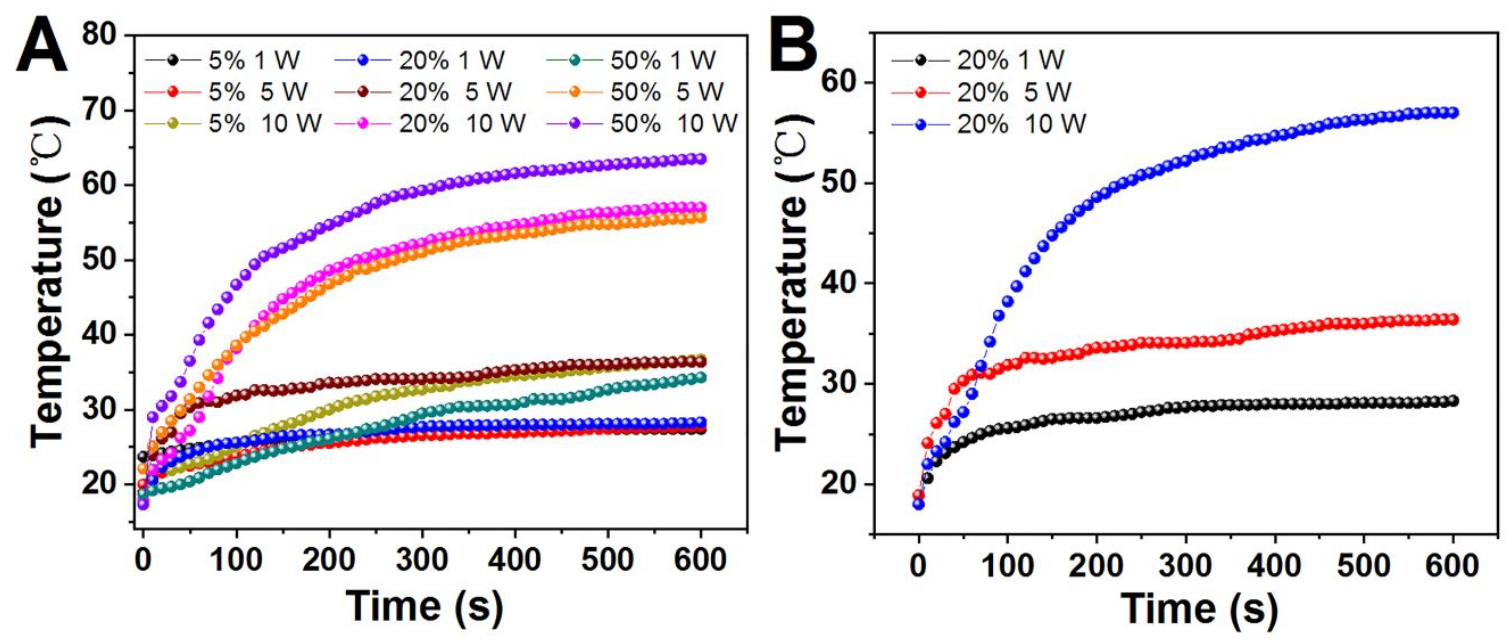

Figure S3. (A) Temperature changes of pork liver tissue under mechanical HIFU with different power and duty cycle. (B) Temperature changes of pork liver tissue under mechanical HIFU (20\% duty cycle) with different power. 


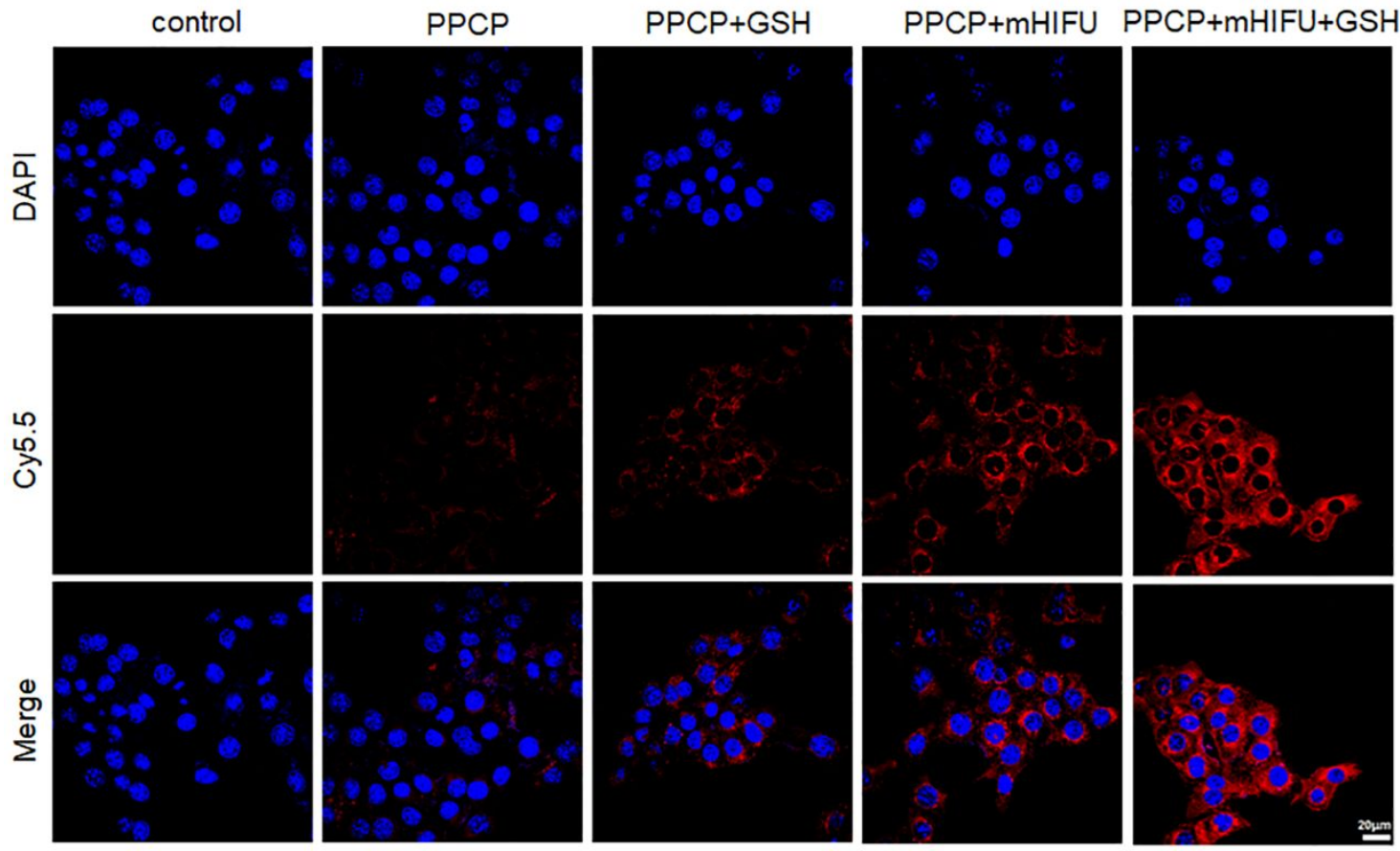

Figure S4. Cellular uptake observations at $4 \mathrm{~h}$ by CLSM. 


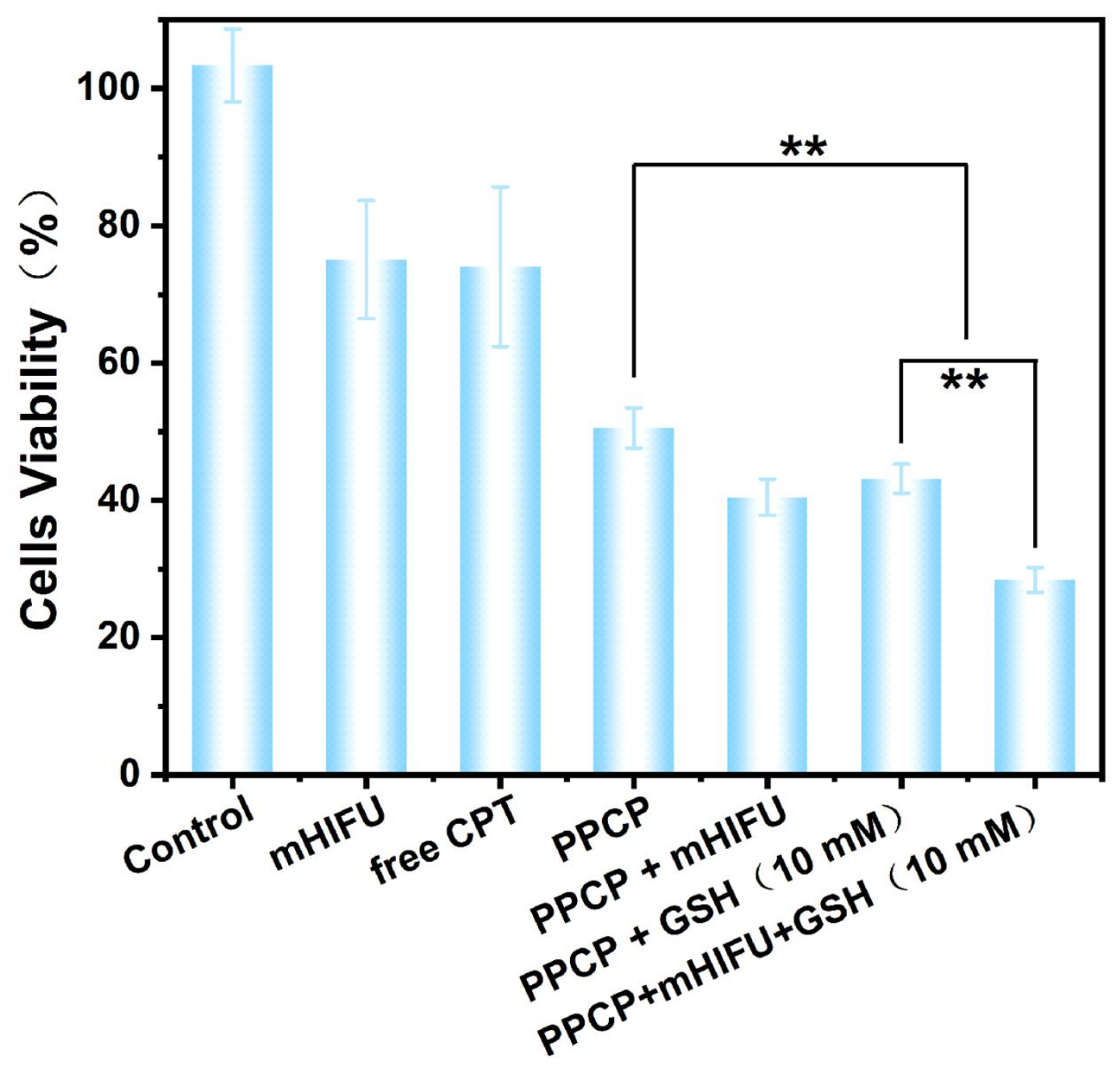

Figure S5. MTT assays under the conditions of free CPT, mHIFU or PPCP NDs exposure to $10 \mathrm{mM}$ of GSH and/or mHIFU irradiation (3.5 MHz, 20\% Duty Cycle, $5 \mathrm{~W}$ ), ${ }^{*} \mathrm{p}<0.05$, $* * \mathrm{p}<0.01$, data are shown as mean $\pm \mathrm{SD}, \mathrm{n}=3$. 


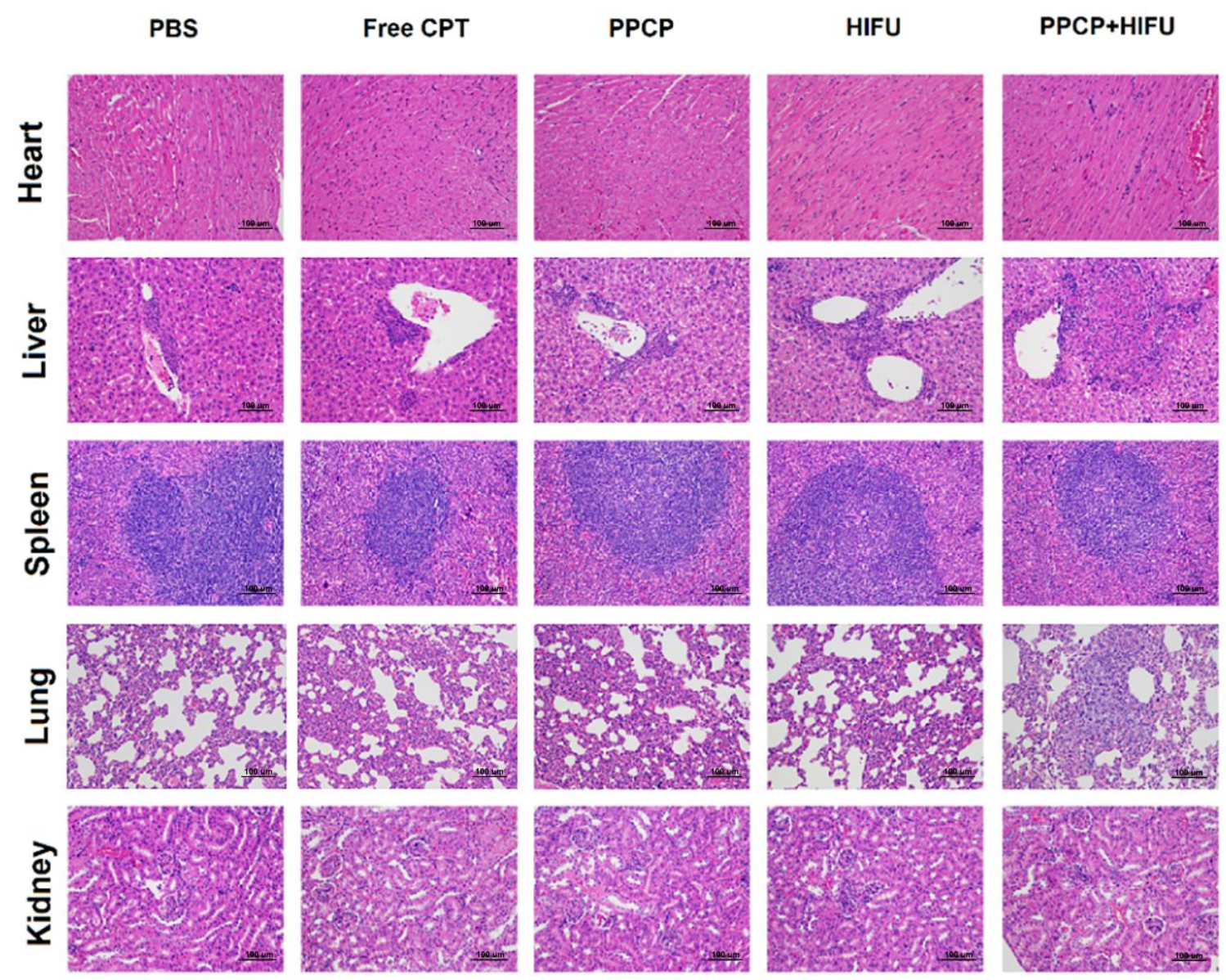

Figure S6. H\&E stained tissues were harvested from the mice of different groups. 

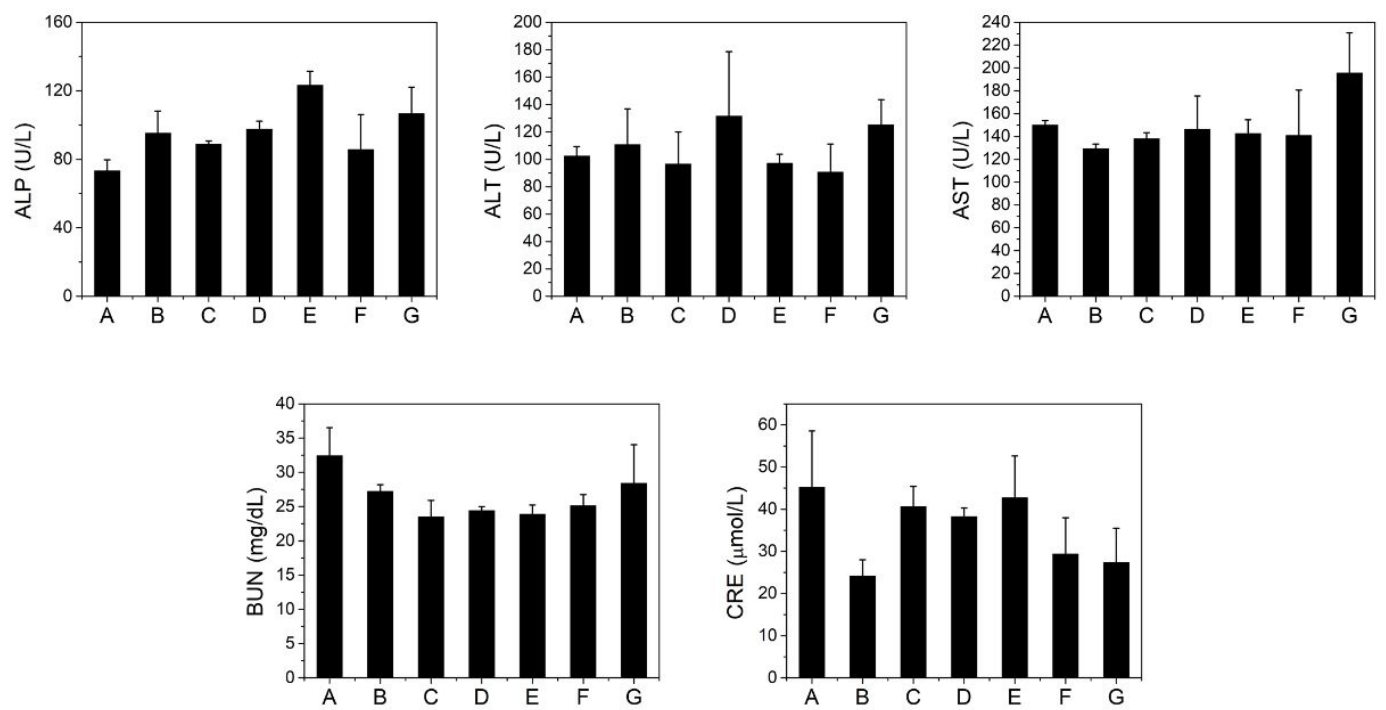

Figure S7. Blood biochemistry data of female BALB/c nude mice treated with PBS (A), Free CPT (B), PPCP NVs (C), HIFU (thermal) (D), HIFU (mechanical) (E), PPCP NVs + HIFU (thermal) (F), PPCP NVs + HIFU (mechanical) (G). 